\title{
High harmonic generation in ethylene with infrared pulses
}

\author{
C. Trallero-Herrero ${ }^{\text {a }}$, B.E. Schmidt ${ }^{\text {a,b }}$, A.D. Shiner ${ }^{c}$, P. Lassonde ${ }^{\text {b }}$, É. Bisson ${ }^{\text {b }}$ J.-C. Kieffer ${ }^{\text {b }}$, \\ P.B. Corkum ${ }^{\mathrm{a}}$, D.M. Villeneuve ${ }^{\mathrm{c}}$, F. Légaré ${ }^{\mathrm{b}, *}$ \\ a Joint Laboratory for Atto-Second Science, University of Ottawa/NRC, 100 Sussex Dr., Ottawa, Ontario, Canada K1A OR6 \\ ${ }^{\mathrm{b}}$ INRS-Énergie et Matériaux, 1650 boul. Lionel-Boulet, C.P. 1020, Varennes (Québec), Canada J3X 1S2 \\ ${ }^{\mathrm{c}}$ National Research Council of Canada, 100 Sussex Dr., Ottawa, Ontario, Canada K1A OR6
}

\section{A R T I C L E I N F O}

\section{Article history:}

Received 6 July 2009

Accepted 9 October 2009

Available online 28 October 2009

\section{Keywords:}

High harmonic generation

Infrared laser

Optical parametric amplifier

\begin{abstract}
A B S T R A C T
We produce harmonics in the molecule ethylene $\left(\mathrm{C}_{2} \mathrm{H}_{4}\right)$ with two different wavelengths and three pulse durations. Due to the low ionization potential $\left(I_{p}=10.5 \mathrm{eV}\right)$ of $\mathrm{C}_{2} \mathrm{H}_{4}$, longer wavelengths are needed to extend the maximum photon energy produced. Our results show that regardless of the strong dependence of the efficiency of the harmonic generation process with wavelength, at $1820 \mathrm{~nm}$ the cutoff observed is larger than that obtained with $800 \mathrm{~nm}, 15 \mathrm{fs}$ pulses. We obtain harmonics with energies exceeding $70 \mathrm{eV}$ at $9.0 \times 10^{13} \mathrm{~W} / \mathrm{cm}^{2}$ with a $73 \mathrm{fs} 1820 \mathrm{~nm}$ pulse. Under these circumstances, the limiting factor for the further extension of the cutoff seems to be the photoionization cross section of the molecule.
\end{abstract}

() 2009 Elsevier B.V. All rights reserved.

\section{Introduction}

Through the process of high harmonic generation (HHG), new possibilities in chemical physics have arisen. In many aspects, this process can be seen as a new type of atomic and molecular spectroscopy which has lead to the full reconstruction of a molecular orbital [1]. Tomography of molecular orbitals has yet to overcome many hurdles and for this reason alternative approaches has been proposed [2]. However, one aspect which can be improved relatively easy is to increase the spatial resolution by increasing the driving field's wavelength [3]. In the strong field approximation (SFA), the generation of harmonics can be explained in a simple, quasi-classical three step model; Ionization, acceleration under the influence of the electric field, and recombination [4]. Once the electron recombines it emits a photon with energy up to,

$E_{p}=I_{p}+3.17 U_{p}$,

with $I_{p}$ being the ionization potential, and $U_{p}$ the ponderomotive energy. Thus, the final step in HHG can be seen as the inverse process to photoionization. Therefore, the amplitude of the harmonics obtained in a single shot measurement can contain information about the inverse process; a bound electron absorbing a photon of energy $\varepsilon$ and going to the continuum $[5,1,6]$. This relationship can be exploited to retrieve, in a table top experiment, information about the photoionization cross section of molecules and atoms in the XUV regime. As mentioned above, the main limitation to this approach is the fact that the maximum XUV photon energy is lim-

\footnotetext{
* Corresponding author. Tel.: +1 450929 8271; fax: +1 4509298201.

E-mail address: legare@emt.inrs.ca (F. Légaré).
}

ited by the ponderomotive energy. Since $U_{p} \propto \lambda^{2}$, with $\lambda$ the center wavelength of the driving field, it is expected that by using longer wavelengths the cutoff of the HHG would extend. Furthermore, at high intensities, molecules with low $I_{p}$ ionize more easily at shorter wavelengths compared to longer wavelengths. The latter has implications for the generation of harmonics due to the fact that, after total ionization, the HHG process in the neutral molecule will cease since there is no interference between the continuum and the ground state and the amplitude of the third step (recombination) is zero. Unfortunately, it was predicted theoretically [7-9] and recently shown experimentally [10] that the efficiency of this process decreases exponentially with wavelength $\mathrm{HHG} \propto \lambda^{x}$ with $x \sim-5$ to -6 . This result means that, in principle, one of the more "trivial" ways of extending the cutoff is not a viable one. We should emphasize that this unfavourable dependence of HHG with wavelength is only for a thin jet geometry where phase matching does not play a crucial role. Recent results show that in a waveguide geometry the decrease in XUV brightness with wavelength can be overcome by enhancing phase matching conditions [11].

In the case of molecular imaging, the importance of extending this cutoff lies in that, by expanding the spectrum of the XUV photons, to all possible non-zero values of the dipole moment of the ground state, we can gather enough information to image a molecular orbital [1]. Spatially this means that the photons emitted in the recombination step must have a wavelength small compared to the size of the molecular orbital features. By no means this is the only reason why a higher cutoff is desired and recently some groups have reported on the generation of harmonics with long wavelengths geared towards other applications [11-13]. In this paper, we report of a HHG spectra obtained in the molecule ethylene 
$\left(\mathrm{C}_{2} \mathrm{H}_{4}\right)$ that expands to $75 \mathrm{eV}$, covering an energy range in which the photoionization cross section of $\mathrm{C}_{2} \mathrm{H}_{4}$ decreases by a factor of 60 [14].

\section{Experimental setup}

The experiment was conducted at the Advanced Laser Light Source (ALLS) using two laser sources. A schematic of the experiment is shown in Fig. 1. For $800 \mathrm{~nm}$ experiments we used a $5 \mathrm{kHz} 500 \mu \mathrm{J}, 35 \mathrm{fs}$ Ti:sapphire laser system. At this wavelength we used pulses of durations $35 \mathrm{fs}$ and $15 \mathrm{fs}$. Pulse shortening was achieved via spectral broadening in Ar at atmospheric pressure in a $250 \mu \mathrm{m}$ diameter hollow-core fiber. Subsequent to the capillary, compensation of material dispersion and pulse compression down to 15 fs was achieved by five round trips on chirped mirrors. The same fiber can be used under vacuum, to avoid self-phase modulation and produce spatially filtered $35 \mathrm{fs}$ pulses after compensating for the second order dispersion induced by the chirped mirrors (CM in Fig. 1) with the amplifier grating compressor. Pulse durations at this wavelength were measured with an auto-correlator. For the $1820 \mathrm{~nm}$ wavelength experiment, we utilized a $100 \mathrm{~Hz}$, $100 \mathrm{~mJ}, 45 \mathrm{fs}$, Ti:sapphire laser system. Laser pulses of $7 \mathrm{~mJ}$ at $800 \mathrm{~nm}$ were directed to an optical parametric amplifier (HE-TOPAS from Light Conversion) and frequency shifted to $1820 \mathrm{~nm}$ idler pulses. The pulse energy and temporal duration were measured to be about $800 \mu \mathrm{J}$ and $73 \mathrm{fs}$. Pulse duration was measured using a second harmonic generation frequency resolved optical gating apparatus. Spatial filtering was performed using a hollow-core fiber ( $400 \mu \mathrm{m}$ diameter, $1 \mathrm{~m}$ long). As in the case of $800 \mathrm{~nm}$, the fiber was under vacuum to avoid self-phase modulation. We verified that the input and output spectra remained identical.

For both wavelengths we focused into the fiber with a $f=1000 \mathrm{~mm}$ plano-convex lens. At the output, the laser beam was collimated with an $R=2000 \mathrm{~mm}$ concave silver mirror. Spatially filtering the beams was crucial in comparing different lasers since it has been observed that it can greatly increase the harmonic efficiency [15-17]. The filtered beam was focused by an $R=500 \mathrm{~mm}$ concave silver mirror into a thin pulsed gas jet with a $500 \mu \mathrm{m}$ aperture backed with a pressure of roughly $3 \mathrm{~atm}$. The jet was mounted on a three-axis manipulator for adjustment of its position. The laser energy could be continuously varied using a half-waveplate in front of the fiber, and a Germanium mirror at Brewster's angle acting as a polarizer ( $\mathrm{P}$ in Fig. 1). The position of the laser focus relative to the center of the jet was determined using the signal of an ion detector, consisting of a mesh biased at

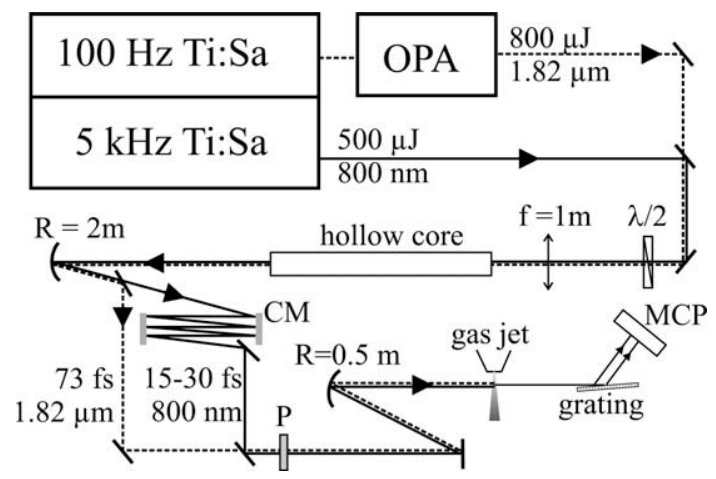

Fig. 1. Optical setup used. Solid line is the $800 \mathrm{~nm}$ beam path. The dashed lines show the path for the $1820 \mathrm{~nm}$ beam. The diameter of the hollow core fiber was $250 \mu \mathrm{m}$ for $800 \mathrm{~nm}$ and $400 \mu \mathrm{m}$ for the $1820 \mathrm{~nm}$ wavelength. For the longer $800 \mathrm{~nm}$ pulse ( $35 \mathrm{fs}$ ), the beam follows the same path (solid line) shown here and the dispersion introduced by the chirped mirrors (CM) was compensated by means of the compressor grating. For curved mirrors we quote the radius of curvature $R=2 \times f$.
$-500 \mathrm{~V}$, a high frequency decoupling circuit and an amplifier connected to a digital oscilloscope. The ion detector assembly was held a few inches below the gas nozzle. The harmonic emission, collimated in the direction of the laser beam was dispersed by a variable groove density concave grating (Hitachi 001-0266) and imaged onto a chevron microchannel plate (MCP) sensitive to wavelengths below $100 \mathrm{~nm}$.

\section{Results}

Since the focusing conditions vary drastically from $800 \mathrm{~nm}$ to $1820 \mathrm{~nm}$, we could not rely on monitoring the energy and pulse durations as a measure of laser intensity. For intensity calibration, we used the space averaged ionization yield with respect to pulse energy in atomic Xe, measured in a separate experiment under the same conditions as $\mathrm{C}_{2} \mathrm{H}_{4}$. We then fit to this curve an ionization model which gives the intensity for a given pulse energy and duration. This procedure is outlined in [10] and [18]. We used the Yudin-Ivanov nonadiabatic ionization model [19] since it is capable of calculating ionization rates at all regimes, from multiphoton to pure tunnelling. By using an atomic specie for the intensity calibration we avoid many of the complications present in modeling molecular ionization. The intensity calibration for the $800 \mathrm{~nm}$, 15 fs data was done in a different way. We start with the intensity calibration at the same wavelength, $35 \mathrm{fs}$ pulses, using the ionization yield as described above. We then find a pulse energy where the HHG spectra from the $800 \mathrm{~nm}, 15 \mathrm{fs}$ pulse has the same cutoff as the $15 \mathrm{fs}, 800 \mathrm{~nm}$ pulse. Since the intensity for the $35 \mathrm{fs}$ case is known and the cutoff does not depend on pulse duration, but only on peak intensity (for the same wavelength and $I_{p}$ ), we then know that the 15 fs pulse has the same peak intensity. This allows to obtain the proportionality factor between energy and intensity which we extrapolate for all energies. The accuracy of this process is then corroborated using Eq. (1) by observing the position of the cutoff at different peak intensities. All the spectra shown in this paper have been corrected by the transformation Jacobian from pixels in the $\mathrm{MCP} /$ camera to photon energy $J=\frac{d_{\text {pixel }}}{d \varepsilon}$, with $\varepsilon$ the photon energy. Since the grating dispersion is almost linear with wavelength, the Jacobian $J(\varepsilon) \approx \frac{1}{\varepsilon^{2}}$. This correction becomes important for high energies where the resolution of the $\mathrm{MCP} / \mathrm{eV}$ is lower and different energies pile up in a single pixel, thus giving an unrealistic harmonic yield at higher energies. The XUV photon energy is calculated using the grating equation [20]. As the cutoff we used the highest harmonic that is clearly visible above the noise floor.

We produced harmonics in an isotropic sample of $\mathrm{C}_{2} \mathrm{H}_{4}$. This molecule has $I_{p}=10.51 \mathrm{eV}$ which is a typical value for organic compounds. Harmonics where produced with three different laser pulses. Firstly $800 \mathrm{~nm} 35 \mathrm{fs}$, representing a typical pulse out of a commercial Ti:Sa system. Secondly, we used an $800 \mathrm{~nm}, 15 \mathrm{fs}$ pulse, obtained as explained in the previous section, and finally, we used $1820 \mathrm{~nm}$ light from a HE-TOPAS. Fig. 2 shows harmonics produced with $800 \mathrm{~nm}, 35 \mathrm{fs}$ pulses and intensity $I \approx 9.8 \times$ $10^{13} \mathrm{~W} / \mathrm{cm}^{2}$ focused in $\mathrm{C}_{2} \mathrm{H}_{4}$. As expected, the cutoff obtained is around $30 \mathrm{eV}$, confirming that at the focus there is good phase matching. This is achieved by using a $250 \mu \mathrm{m}$ fiber to spatially filter the otherwise Gaussian beam; a technique that has proven to increase the HHG yield [15-17]. In order to extend the cutoff, another common technique is to use ultrashort short pulses obtained after broadening the spectrum via self-phase modulation in a capillary. The cutoff extension in this case is two fold. Firstly because of the mentioned mode filtering, and secondly because short pulses allow for atoms or molecules to experience higher intensities before ionization saturation occurs. In Fig. 3, we show harmonics obtained with $800 \mathrm{~nm}, 15 \mathrm{fs}$ pulses in ethylene at the following two intensities $I \approx 9.8 \times 10^{13} \mathrm{~W} / \mathrm{cm}^{2}$ (black curve) and 


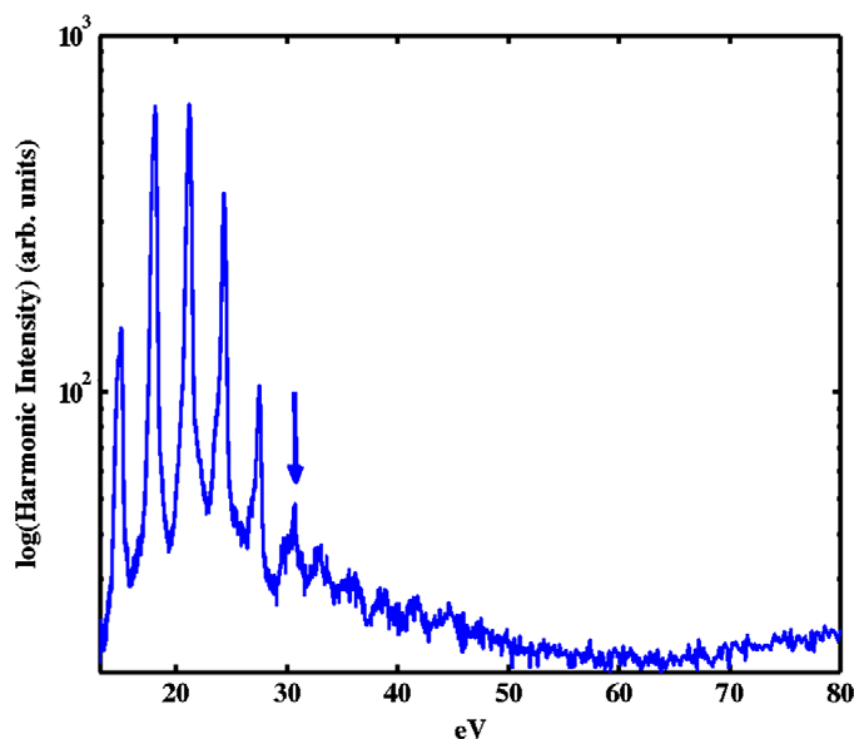

Fig. 2. Harmonics generated with $800 \mathrm{~nm} 35$ fs pulses in $\mathrm{C}_{2} \mathrm{H}_{4}$ with intensity $I \approx 9.8 \times 10^{13} \mathrm{~W} / \mathrm{cm}^{2}$. The arrow points the spectrum's cutoff determined as the highest harmonic above the noise floor.

$I \approx 1.45 \times 10^{14} \mathrm{~W} / \mathrm{cm}^{2}$ (red curve). Intensities obtained by means of the semiclassical cutoff law are $I_{c}=1.0 \times 10^{14} \mathrm{~W} / \mathrm{cm}^{2}$ for a cutoff of $30.4 \mathrm{eV}$ and $I \approx 1.5 \times 10^{14} \mathrm{~W} / \mathrm{cm}^{2}$ for a cutoff of $40 \mathrm{eV}$. The close agreement between the calculated semiclassical and observed cutoff tells us that for these intensities HHG in ethylene is still following the cutoff law. This is a strong indication that the ionization step is still not saturated. By comparing this result to Fig. 2 we observe that the cutoff is the same when both pulses have similar intensities, as it should be according to Eq. (1) but now we can extend the maximum energy of the XUV photons by increasing the intensity of the driving field. Even though $15 \mathrm{fs}$ is a very modest pulse duration compared to shorter durations obtained nowadays, it does represent a fairly easy attainable one. The spectrum at the highest intensity agrees with that obtained in [21] for similar values of the intensity.

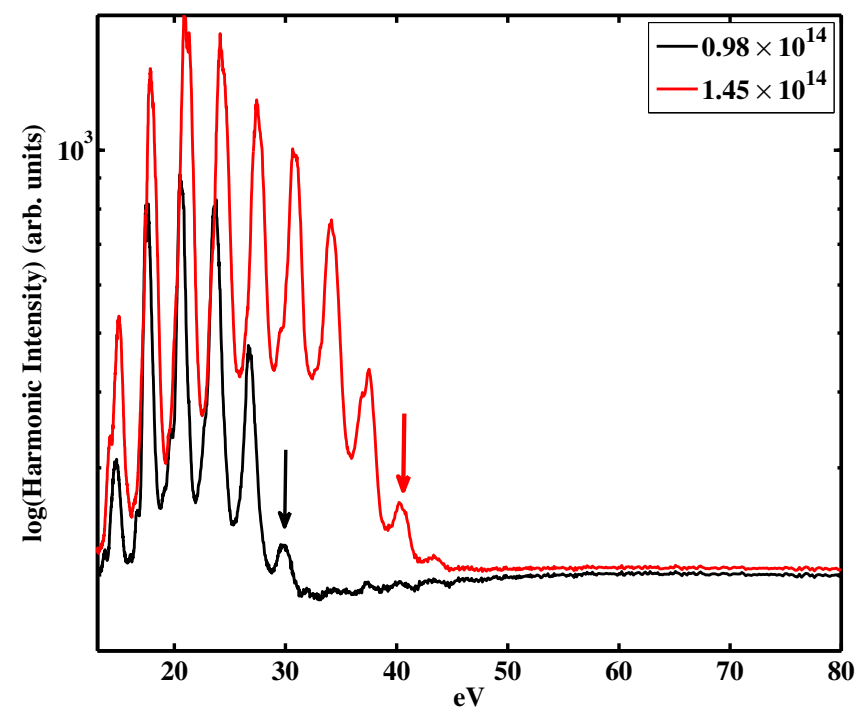

Fig. 3. Harmonics generated with $800 \mathrm{~nm} 15$ fs pulses in $\mathrm{C}_{2} \mathrm{H}_{4}$ with intensities $I \approx 9.8 \times 10^{13} \mathrm{~W} / \mathrm{cm}^{2}$ (black) and $I \approx 1.45 \times 10^{14} \mathrm{~W} / \mathrm{cm}^{2}$ (red). The arrows points the spectrum's cutoff determined as the highest harmonic above the noise floor. (For interpretation of the references to colour in this figure legend, the reader is referred to the web version of this article.)
Finally, we proceed to use the idler beam out of the HE-TOPAS at $1820 \mathrm{~nm}$. Although we obtained $800 \mu \mathrm{J}$ out of the HE-TOPAS, at the chamber only $250 \mu \mathrm{J}$ remained. These losses came from absorption in air, and poor reflectivity of the mirrors, but mainly at the fiber, where the transmission efficiency is around 50\%. However, it was shown that despite great losses in this filtering process, HHG can benefit greatly from it. Ref. [15] quotes enhancements of a factor of 10 at $1400 \mathrm{~nm}$, using the signal from the same HE-TOPAS. For the idler, we expect this enhancement factor to be even larger due a poorer mode quality. Fig. 4 shows harmonics obtained with $1820 \mathrm{~nm}, 73 \mathrm{fs}$ and $I \approx 9.0 \times 10^{13} \mathrm{~W} / \mathrm{cm}^{2}$ pulses in ethylene. As previously mentioned, the strong power law dependence of harmonic efficiency predicts that at this wavelength the obtained spectra is roughly $(1820 / 800)^{6} \sim 141$ times less bright than at $800 \mathrm{~nm}$. To compensate for this we increased the MCP gain while insuring that the detector is still in the linear regime for amplification. Despite the very unfavourable HHG efficiency at this wavelength we obtained photon energies up to $75 \mathrm{eV}$. However, the cutoff law Eq. (1) predicts a maximum energy of $94 \mathrm{eV}$. One might argue that a reason for discrepancy is a difference in phase matching. We argue this is not the case as we spatially filtered all beams and the $800 \mathrm{~nm}$ produced harmonics follow Eq. (1) very closely. Another cause could be that since we are using a very long pulse the sample is totally ionized and therefore, the HHG process is saturated. Still, results shown in [21] and in this article for $800 \mathrm{~nm}$ predict that fairly high intensities are needed to saturate the first ionization in this molecule. Also, a large error in the intensity calibration is a possible reason for the discrepancy. At this point we have no quantitative measurement on the error for the intensity calibration method used, however, it does work remarkably well at other wavelengths where the cutoff is not as large. Furthermore, if the intensity was $20-25 \%$ smaller, the ionization rate and the harmonic process, would drop drastically due to the exponential dependence that tunnel ionization has with field strength. If this is indeed the case, the large discrepancy between the predicted and observed cutoff at this wavelength might be due to effects other than just ionization saturation or a large error in the estimation of the intensity.

According to the SFA model, there are three steps in the generation of harmonics; ionization, acceleration in the field, and recombination. The last step involves the emission of a XUV photon and can be described by the recombination dipole moment,

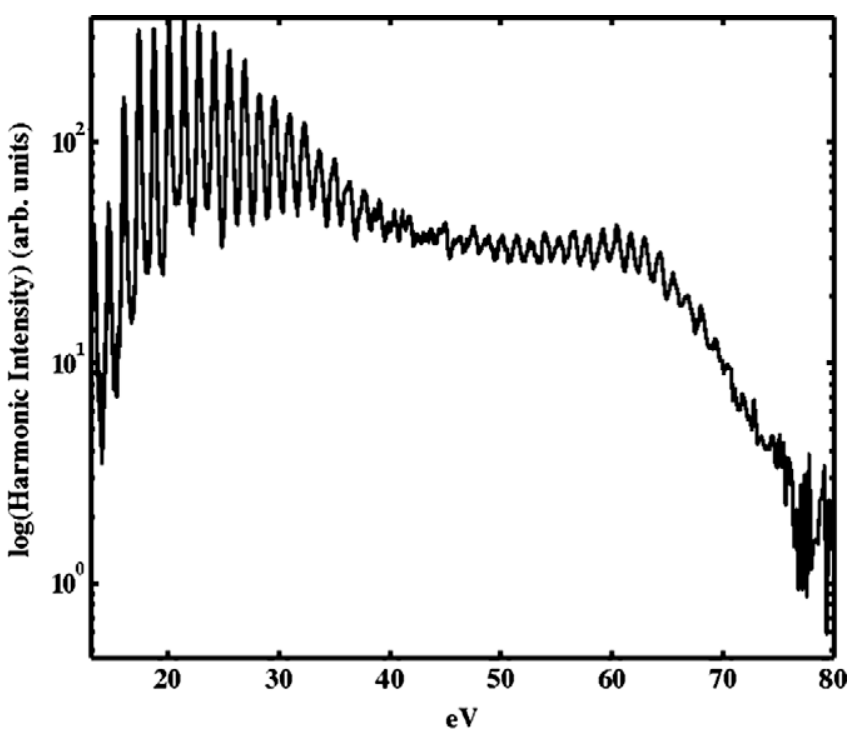

Fig. 4. Harmonics generated with $1820 \mathrm{~nm} 80 \mathrm{fs}$ pulses in $\mathrm{C}_{2} \mathrm{H}_{4}$ with intensity $I \approx 9.0 \times 10^{13} \mathrm{~W} / \mathrm{cm}^{2}$. 
$\left\langle\Phi(v)|x| \Psi_{g}\right\rangle$, where $\Phi(v)$ is the continuum wave function with electron's velocity $v$, and $\Psi_{g}$ is the electron's ground state. This dipole moment can also describe the inverse process, that is, ionization through a photon with energy $\varepsilon=I_{p}+K(v)$, producing an electron with kinetic energy $K(v)$. HHG requires a non-zero photoionization cross section (dipole moment) to produce a photon of energy $\varepsilon$. In $\mathrm{C}_{2} \mathrm{H}_{4}$, this matrix element approaches zero, decreasing by a factor of roughly 60 from $20 \mathrm{eV}$ to $100 \mathrm{eV}[14,22]$. This will reduce the yield at high photon energies when compared to noble gases.

\section{Concluding remarks}

We have generated harmonics in ethylene with the following three different pulses; $800 \mathrm{~nm}$ with durations of $35 \mathrm{fs}$ and $15 \mathrm{fs}$, and $1820 \mathrm{~nm}$ with a duration of $73 \mathrm{fs}$. At such a long wavelength, harmonics can be measured by spatially filtering the idler beam out of a HE-TOPAS. We find that due to the dependence of the cutoff with $\lambda^{2}$, the cutoff extends reaching energies up to $75 \mathrm{eV}$. This value differs largely from the $94 \mathrm{eV}$ one predicted by theory. Since we use the same spatial filter for both the $800 \mathrm{~nm}$ and $1820 \mathrm{~nm}$ experiments, and the former follows the theoretical prediction Eq. (1) quite closely, we argue that phase matching should not be the case for the large discrepancy observed in the latter. Furthermore, we observe continually increasing harmonics with intensity up to $1.45 \times 10^{14} \mathrm{~W} / \mathrm{cm}^{2}$, at $800 \mathrm{~nm}$ which is an intensity much larger than the maximum intensity used at $1820 \mathrm{~nm}$, thus eliminating the possibility of ionization saturation. For this case $(1820 \mathrm{~nm})$, the limiting factor for the maximum photon energy obtainable is likely the photoionization cross section which is related to HHG through the ionization/recombination dipole moment. At first, this could be seen as a limitation, however, it might open the possibility to study photoionization of molecules in the XUV regime, using table top optics. Perhaps of more relevance to the atomic and molecular physics community is the conclusion that we can expand the spectrum of the XUV photons, to all possible non-zero values of the dipole moment of the ground state, providing enough information to, in principle, image molecular orbitals [1]. Further experiments should still be done to test our findings. We plan to use higher peak intensities to observe a better contrast between the observed and calculated cutoff. This can be achieved by using higher pulse energies or pulse compression techniques, both of which we are currently implementing based on recent developments $[23,24]$.

\section{Acknowledgements}

The authors would like to thank the technical staff at ALLS for their dedicated support. We also acknowledge the support of the Canadian Foundation for Innovation, the Canadian Institute for Photonic Innovations, The Natural Sciences and Engineering Research Council of Canada, the Fonds Québécois de la Recherche sur la Nature et les Technologies, and the Air Force Office of Scientific Research (AFOSR).

\section{References}

[1] J. Itatani, J. Levesque, D. Zeidler, H. Niikura, H. Pepin, J.C. Kleffer, P.B. Corkum, D.M. Villeneuve, Tomographic imaging of molecular orbitals, Nature 432 (2004) 867.
[2] W. Boutu, S. Haessler, H. Merdji, P. Breger, G. Waters, M. Stankiewicz, L.J. Frasinski, R. Taieb, J. Caillat, A. Maquet, P. Monchicourt, B. Carre, P. Salieres, Coherent control of attosecond emission from aligned molecules, Nat. Phys. 4 (7) (2008) 545.

[3] V.-H. Le, A.-T. Le, R.-H. Xie, C.D. Lin, Theoretical analysis of dynamic chemical imaging with lasers using high-order harmonic generation, Phys. Rev. A 76 (1) (2007) 013414.

[4] M. Lewenstein, P. Balcou, M.Y. Ivanov, A.L. L'Huillier, P.B. Corkum, Theory of high-harmonic generation by low frequency laser fields, Phys. Rev. A 49 (1994) 2117.

[5] T. Brabec, M.Y. Ivanov, P.B. Corkum, Coulomb focusing in intense field atomic processes, Phys. Rev. A 54 (4) (1996) R2551.

[6] M.V. Frolov, N.L. Manakov, T.S. Sarantseva, M.Y. Emelin, M.Y. Ryabikin, A.F. Starace, Analytic description of the high-energy plateau in harmonic generation by atoms: can the harmonic power increase with increasing laser wavelengths?, Phys Rev. Lett. 102 (24) (2009) 243901.

[7] J. Tate, T. Auguste, H.G. Muller, P. Salières, P. Agostini, L.F. DiMauro, Scaling of wave-packet dynamics in an intense midinfrared field, Phys. Rev. Lett. 98 (1) (2007) 013901.

[8] K. Schiessl, K.L. Ishikawa, E. Persson, J. Burgdörfer, Quantum path interference in the wavelength dependence of high-harmonic generation, Phys. Rev. Lett. 99 (25) (2007) 253903.

[9] M.V. Frolov, N.L. Manakov, A.F. Starace, Wavelength scaling of high-harmonic yield: threshold phenomena and bound state symmetry dependence, Phys. Rev. Lett. 100 (17) (2008) 173001.

[10] A.D. Shiner, C. Trallero-Herrero, N. Kajumba, H.-C. Bandulet, D. Comtois, F Légaré, M. Giguère, J.-C. Kieffer, P.B. Corkum, D.M. Villeneuve, Wavelength scaling of high harmonic generation efficiency, Phys. Rev. Lett. 103 (7) (2009) 073902.

[11] T. Popmintchev, M.-C. Chen, A. Bahabad, M. Gerrity, P. Sidorenko, O. Cohen, I.P. Christov, M.M. Murnane, H.C. Kapteyn, Phase matching of high harmonic generation in the soft and hard X-ray regions of the spectrum, P.N.A.S. 106 (26) (2009) 10516.

[12] P. Colosimo, G. Doumy, C.I. Blaga, J. Wheeler, C. Hauri, F. Catoire, J. Tate, R. Chirla, A.M. March, G.G. Paulus, H.G. Muller, P. Agostini, L.F. DiMauro, Scaling strong-field interactions towards the classical limit, Nat. Phys. 4 (5) (2008) 386.

[13] G. Doumy, J. Wheeler, C. Roedig, R. Chirla, P. Agostini, L.F. DiMauro, Attosecond synchronization of high-order harmonics from midinfrared drivers, Phys. Rev. Lett. 102 (9) (2009) 093002.

[14] J. Berkowitz, Atomic and Molecular Photoabsorption: Absolute Total Cross Sections, Academic Press, 1979.

[15] H.-C. Bandulet, D. Comtois, A.D. Shiner, C. Trallero-Herrero, N. Kajumba, T Ozaki, P.B. Corkum, D.M. Villeneuve, J.-C. Kieffer, F. Legare, High harmonic generation with a spatially filtered optical parametric amplifier, J. Phys. B 41 (24) (2008) 245602 (7pp.).

[16] M. Nisoli, E. Priori, G. Sansone, S. Stagira, G. Cerullo, S. De Silvestri, C. Altucci, R Bruzzese, C. de Lisio, P. Villoresi, L. Poletto, M. Pascolini, G. Tondello, Highbrightness high-order harmonic generation by truncated bessel beams in the sub-10-fs regime, Phys. Rev. Lett. 88 (3) (2002) 033902.

[17] C. Altucci, R. Bruzzese, C. de Lisio, M. Nisoli, E. Priori, S. Stagira, M. Pascolini, L. Poletto, P. Villoresi, V. Tosa, K. Midorikawa, Phase-matching analysis of highorder harmonics generated by truncated bessel beams in the sub-10-fs regime, Phys. Rev. A 68 (3) (2003) 033806.

[18] S. Hankin, D. Villeneuve, P. Corkum, D. Rayner, Intense-field laser ionization rates in atoms and molecules, Phys. Rev. A 64 (2001) 013405.

[19] G.L. Yudin, M.Y. Ivanov, Nonadiabatic tunnel ionization: looking inside a laser cycle, Phys. Rev. A 64 (1) (2001) 013409.

[20] T. Kita, T. Harada, N. Nakano, H. Kuroda, Mechanically ruled aberrationcorrected concave gratings for a flat-field grazing-incidence spectrograph, Appl. Opt. 22 (4) (1983) 512.

[21] N. Kajumba, R. Torres, J.G. Underwood, J.S. Robinson, S. Baker, J.W.G. Tisch, R. de Nalda, W.A. Bryan, R. Velotta, C. Altucci, I. Procino, I.C.E. Turcu, J.P. Marangos, Measurement of electronic structure from high harmonic generation in non-adiabatically aligned polyatomic molecules, New J. Phys. 10 (2) (2008) 025008 (17pp.).

[22] G. Cooper, T.N. Olney, C. Brion, Absolute UV and soft X-ray photoabsorption of ethylene by high resolution dipole (e,e) spectroscopy, Chem. Phys. 194 (1) (1995) 175 .

[23] E.J. Takahashi, T. Kanai, Y. Nabekawa, K. Midorikawa, $10 \mathrm{~mJ}$ class femtosecond optical parametric amplifier for generating soft X-ray harmonics, Appl. Phys. Lett. 93 (4) (2008) 041111.

[24] M. Giguère, B.E. Schmidt, A.D. Shiner, M.-A. Houle, H.C. Bandulet, G. Tempea, D.M. Villeneuve, J.-C. Kieffer, F. Légaré, Pulse compression of submillijoule fewoptical-cycle infrared laser pulses using chirped mirrors, Opt. Lett. 34 (12) (2009) 1894. 